Journal of Diagnostic Pathology 2011 (6); 1: 44-46

\title{
Isolated intracranial Rosai-Dorfman disease
}

Siriweera $\mathrm{EH}^{1}$, Ratnatunga $\mathrm{NVI}^{2}$, Wickramasinghe $\mathrm{R}^{3}$, Kumarasinghe $\mathrm{N}^{4}$

\section{Case Report}

A 54 year old lady presented with right sided focal seizures and faintishness of one year duration. She also complained of episodic headaches, short term memory impairment, dysphasia and impaired near vision. Constitutional symptoms such as fever, night sweats and weight loss were absent.

General and system examination were unremarkable. Pallor,lymphadenopathyor hepatosplenomegaly were not present. Despite many neurological symptoms the nervous system examination was normal.Routine haematology and serology laboratory investigations yielded normal results. Magnetic resonance imaging (MRI) of the brain revealed a well defined dural based mass lesion in the left parieto-occipital area. After gadolinium diethylenetriamine pentaacetic acid administration, vivid enhancement of the lesion and enhancement of the dural tail were noted. Associated gross perilesional white matter oedema and inner cranial vault hyperostosis were noted.The radiological opinion suggested a meningioma. A left posteroparietal craniotomy was performed. A tumour like lesion with a dural base spreading along the brain surface was identified and excised totally and sent for histological assessment. The post operative course was uneventful. Histopathological examination of two masses of tissue, largest measuring $2.5 \times 1.5 \times 1.5 \mathrm{~cm}$ revealed brain tissue with overlying meninges.

The meninges were heavily infiltrated by histiocytes intermixed with plasma cells and lymphocytes. The majority of the histiocytes showed lymphophagocytosis / emperipolesis. (Fig 1) Many of the plasma cells displayed Russell's bodies. Meningeal fibrosis was also evident. Immunohistochemical staining with S-100 protein revealed strong cytoplasmic and nuclear positivity in the histiocytes. The histological diagnosis was Rosai-Dorfman disease of the meninges.

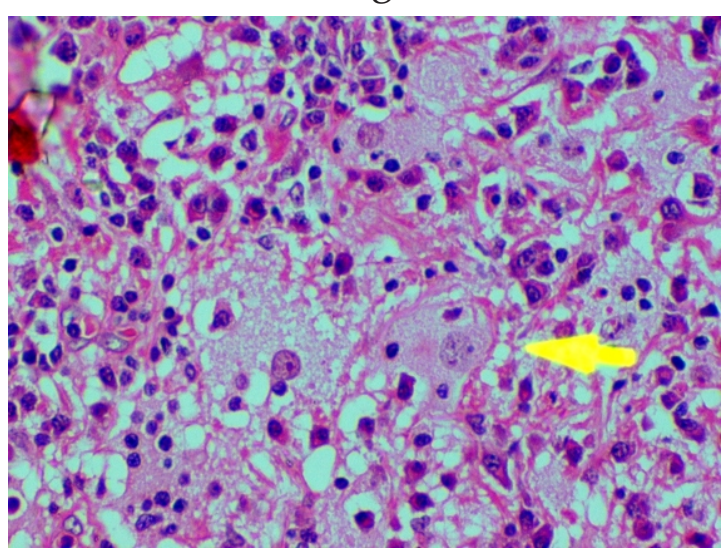

Fig 1. A histiocyte with emperipolesis (arrow) (H\&EX40)

\section{Discussion}

Rosai-Dorfman disease was first described in 1969 as a systemic non neoplastic histioproliferative disease $(1,2)$.Patients usually present with massive painless lymphadenopathy, fever and weight loss. Involvement of an extranodal site including

${ }^{1}$ Lecturer, ${ }^{2}$ Professor of Pathology, Department of Pathology, Faculty of Medicine, University of Peradeniya, Sri Lanka. ${ }^{3}$ Consultant neurosurgeon, ${ }^{4}$ Senior registrar in neurosurgery, Teaching Hospital, Kandy, Sri Lanka.

Correspondance: Siriweera EH, Department of Pathology, Faculty of Medicine, University of Peradeniya, SriLanka.e-mail:s.eranga@yahoo.com 
skin, orbit, respiratory tract, bones, central nervous system and salivary gland is seen in up to $43 \%$ of cases either alone or in association with lymphadenopathy $(1,3)$. Of this central nervous system involvement characterizes less than $5 \%$ of the patient total (3). Approximately 80 cases of central nervous system RosaiDorfman disease have been reported (4). Isolated intracranial involvement is rare (3). Intracranial Rosai-Dorfman disease carries a favourable prognosis after complete resection or corticosteroid therapy (5). Interestingly central nervous system involvement does not adversely affect the prognosis (3). Central nervous system Rosai-Dorfman disease shows a predilection for males, and typically presents during the fourth to fifth decade. Although haematology and serology profiles were normal in this patient, non specific findings such as mild anaemia, elevated erythrocyte sedimentation rate and polyclonal hypergammaglobulinaemia may occur (4). The majority of intracranial lesions as was seen in this case mimic meningiomas presenting as dural based solitary or multiple lesions that enhance strongly following gadolinium administration. As such diagnosis relies on histopathological evaluation.The differential diagnosis in this case was Rosai-Dorfman disease, lymphoplasmacytic meningioma, Langerhan cell histiocytosis and plasma cell granuloma. Sheets of histiocytes with many showing emperipolesis and positivity for S100 protein immunohistochemically confirmed a diagnosis of Rosai-Dorfman disease.

Although emperipolesis is known to be less apparent in extranodal sites, this case demonstrated conspicuous emperipolesis. Lymphoplasmacytic meningioma elicits a pronounced chronic inflammatory reaction but typical meningeal cells were not seen in this case. Langerhan cell histiocytosis may exhibit a xanthomatous appearance but the histiocytes in this case lacked nuclear indentation and longitudinal grooves. In addition emperipolesis is not prominent in Langerhan cell histiocytosis and the cellular infiltrate did not show eosinophils. Hence a Langerhan cell histiocytosis was unlikely. Plasma cell granulomas have a polymorphous cellular infiltrate composed of lymphocytes, plasma cells and histiocytes associated with fibrosis. However emperipolesis and S100 protein positive histiocytes are not a feature of this entity.

To the best of our knowledge this is the first reported case of intracranial Rosai Dorfman disease in Sri Lanka. Although intracranial Rosai Dorfman disease is a rare entity it should be included in the differential diagnosis of a dural mass clinically and radiologically mimicking a meningioma.

\section{References}

1. McPherson CM,BrownsJ, KimAW, DeMoneF.Regression of intracranial Rosai -Dorfman disease following corticosteroid therapy. Journal of Neurosurgery 2006;104: 840-844.

2. Udono H, Fukuyama $\mathrm{K}$, Okamoto $\mathrm{H}$, Tabuchi K. Rosai-Dorfman disease presenting multiple intracranial lesions with unique findings on magnetic resonance imaging. Journal of Neurosurgery 1999; 91:335-339.

3. Andriko JW, Morrison A, Colegial $\mathrm{CH}$, Davis BJ, Jones RV. Rosai-Dorfman disease Isolated to the central nervous system: A report of 11 cases. Modern Pathology 2001; 14(3):172-1. 
4. Kidd DP, Revesz T, Miller NR. RosaiDorfman disease presenting with widespread intracranial and spinal cord involvement. Neurology 2006; 67: 1551-1555.

5. Paulus W, Perry A: Histiocytic tumours. In Louis DN, Ohgaki H, Wiestler OD,Cavenee WK(eds): WHO Classification of tumours of the central nervous system. $4^{\text {th }}$ edition. Lyon: IARC press. 2007; 193-196.

6. Hadjipanyas CG, Bejjani G,Wiley M, Hasegawa T, Maddock M, Kondziolka D.Intracranial Rosai-Dorfman disease treated with microsurgical resection and stereotactic radiosurgery. Journal of Neurosurgery 2003; 98:165-168. 